\title{
Sound Artists:
}

\section{$\underline{E S C ~ l i n k s}$}

JLiat

http://www.jliat.com

Francisco Lopez

http://www.franciscolopez.net

Sally Anne McIntyre

http://radiocegeste.blogspot.com

Jana Winderen

http://www.janawinderen.com

Jan Van Ijken

http://www.janvanijken.com

Chris Watson

http://chriswatson.net

David Dunn

http://earthear.com/dunn.html

Daniel Blinkhorn

https://danielblinkhorn.com

Christina Kubisch

http://www.christinakubisch.de/en/home

Peter Cusack

http://www.arts.ac.uk/research/ual-staff-researchers/a-z/peter-cusack/

Philip Aaberg

https://www.sweetgrassmusic.com

Stephen Barncard, "Foghorns of the Golden Gate"

https://jazzcubednature.bandcamp.com/track/foghorns-of-the-golden-gate

http://mixstream.org

Mallsoft, 猫 シ/ Cat System Corp.

catsystemcorp.bandcamp.com

https://www.facebook.com/catsystemcorp/

Harare Mambos, "Mbuya Nahanda"

https://www.pindula.co.zw/Harare_Mambos 


\section{Environmental issues:}

Invasive species

https://www.fws.gov/invasives/index.html

https://www.invasivespeciesinfo.gov/index.shtml

https://www.naisn.org

Bird-friendly coffee

https://nationalzoo.si.edu/migratory-birds/bird-friendly-coffee

https://www.rainforest-alliance.org

https://www.audubon.org/news/how-choose-bird-friendly-coffee

HABs

http://www.noaa.gov

https://oceanservice.noaa.gov/hazards/hab/

https://www.cdc.gov/habs/index.html

Glacier melt, sea level rise

http://www.climatecentral.org

\section{Wildfires}

https://www.ucsusa.org/global_warming/science_and_impacts/impacts/climate-changedevelopment-patterns-wildfire-costs.html\#.Wykr2y2ZPMU

https://www.epa.gov/climate-indicators/climate-change-indicators-wildfires

Coral bleaching

https://coral.org

https://www.nature.org/ourinitiatives/urgentissues/oceans/coral-reefs/index.htm

\section{Toxic poisoning}

http://www.who.int/en/news-room/fact-sheets/detail/arsenic

https://www.cancer.org/cancer/cancer-causes/arsenic.html

https://www.publicintegrity.org/environment/politics-poison

http://www.panna.org

https://www.beyondpesticides.org

\section{Global warming}

https://www.ucsusa.org/global-warming

https://350.org

https://www.edf.org 